\title{
Spectral classification and variation of Fermi GRBs
}

\section{Istvan I. Racz*}

Eötvös University, RCAES HAS Konkoly Observatory

E-mail: racz@complex.elte.hu

\section{Lajos G. Balázs}

Eötvös University, RCAES HAS Konkoly Observatory

\section{Viktor L. Tóth}

Eötvös University, RCAES HAS Konkoly Observatory

\section{Istvan Horvath}

National University of Public Service

\section{Zsolt Bagoly}

Eötvös University

The Fermi GBM catalog contains general physical quantities of the observed objects and also estimated parameters (peak energy, spectral indices, intensity) from four fitted spectral models (Band, smoothly broken power law, Comptonized, power law) for the peak flux and the fluence. We studied the nature of the errors of the peak flux, the fluence, and duration parameters. We have found a linear correlation between the logarithm of the measured quantities and their error bars. We interpret our results as an indication that the peak flux, fluence and duration follow a Poissonic distribution.

7th Fermi Symposium 2017

15-20 October 2017

Garmisch-Partenkirchen, Germany

\footnotetext{
*Speaker.
} 


\section{Introduction}

Gamma-ray bursts (GRBs) are the most powerful bursts in the far Universe. The timescale of these bursts varies on a wide range - from tens of milliseconds to thousands of seconds [1]. On the other hand, the phenomenological classification scheme would remain an open question. From the 80s we distinguish them on the basis of the burst duration: there are short and long GRBs [2]. It seems that short GRBs are caused by the collision of two massive stars, and hypernovas can explain long GRBs. Nowadays using multi-and uni-variate statistical analysis techniques an intermediate group was found $[3,4,5,6,7,8,9,10,11,12]$. Moreover, more huge structures have been published recently which were created by GRBs: the Hercules-Borealis great wall at $z \approx 2$ $[13,14]$ and the GRB ring at $z \approx 0.8[15,16]$. The genesis of these structures is not known yet.

\section{Data \& Mathematical summary}

The Fermi Gamma-ray Space Telescope (FGST) is a space observatory being used to perform gamma-ray astronomy observations from low Earth orbit since 2008. It has two instruments, the Large Area Telescope (LAT), and the Gamma-ray Burst Monitor (GBM). The GBM is used to study gamma-ray bursts; the data received by its 14 detectors (12 NaI and 2 BGO) are collected by a central Data Processing Unit (DPU) [17]. The database from the Fermi GBM detectors, the FERMIGBRST $^{1}$ catalog $[18,19]$ contains more than 2000 GRBs with several parameters such as position, durations, flux, fluences and spectral properties. These parameters were calculated from the light curves and spectral fitting. In the catalog the best fit model for both the peak flux of the burst ('pflx') and for the entire burst duration ('flnc') is also found. This model can belong in the power law, Comptonized, smoothly broken power law or Band spectral class.

Linear discriminant analysis (LDA) finds $\mathrm{k}-1$ canonical coordinates ( $\mathrm{k}$ being the number of classes) that best separate the categories [20]. These functions - which are called discriminant functions - are uncorrelated and defined in effect, an optimal $\mathrm{k}-1$ space through the $\mathrm{p}$-dimensional cloud of data separates (the projections in that space of) the k groups best. The biggest difference between the groups are shown by the first discriminant function. The subsequent functions show in order of relevance the maximum distance between the groups in the parametric space. We used LDA with Jackknifed Prediction from 'MASS' package in R. Finally, we analyzed the errors of the parameters.

\section{Results}

We studied the relationship between the best fit spectral model and the GRB's model independent physical parameters - duration, flux, fluence - using LDA method [21] on the 'GOOD sample' (published by [18]). Taking into account all the data where the best model was available we found that at least the first discriminate function was significant on both the 'pflx' and 'flnc' spectral types (sign.level $<2.2 \cdot 10^{-16}$ ). From the correlations between the discriminant functions and the input variables, the structure matrix we found that the flux and fluence were important parameters for the separation of the spectral classes but the durations were not. According to LDA the strongest

\footnotetext{
${ }^{1}$ https://heasarc.gsfc.nasa.gov/W3Browse/fermi/fermigbrst.html
} 
separator variable at the 'pflx' type is the Flux and at the 'flnc' type the Fluence. We found that the 'pflx' types could be separated better, even though the 'flnc' spectra has more photons.

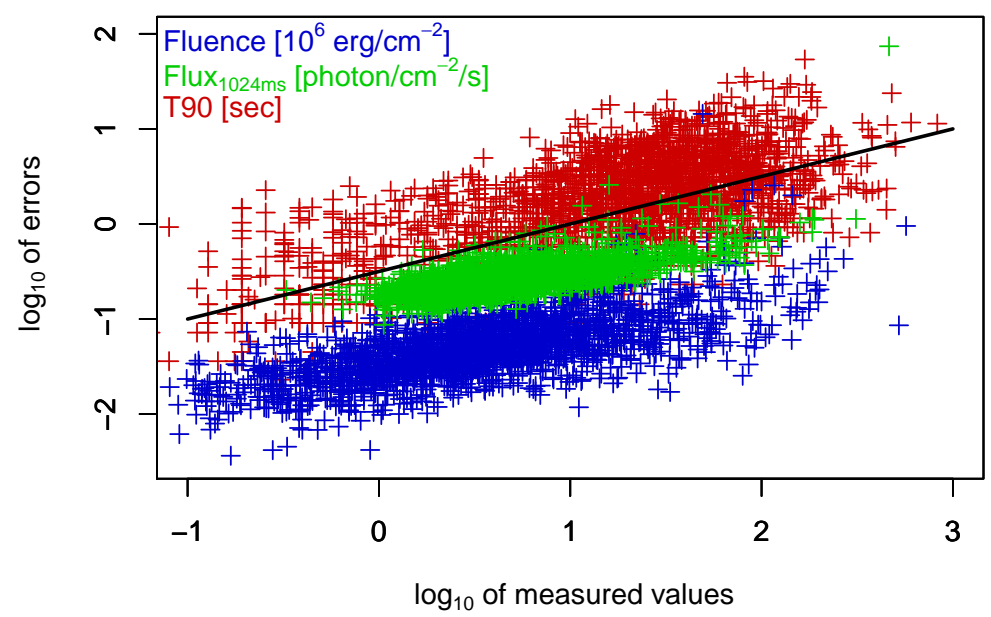

Figure 1: We examined the errors of the main physical parameters and found strong correlation $-0.68 \pm$ 0.037 - between the errors and the measured values on logarithmic scale. The significant slope is $\approx 2: 1$ which can be explained as a result of natural Poissonic noise.

For these anomalies we examined the errors of the main physical parameters because we thought that there is some kind of a systematic error in the examined parameters. Finally, we found a strong a significant correlation $(0.68 \pm 0.037)$ between the errors and the measured values on logarithmic scale Fig. 1. The significance was less than $10^{-5}$. The property of the Poissonic distribution is that its expected value and its variance is equal [22], from which it follows that the standard deviation equals the square of the signal. The slope of this correlation was found at $\approx 0.5$ which can be explained as a result of natural Poissonic noise.

\section{Summary}

We found that there is a strong linear correlation between the Fermi GBM parameters and the errors of the main physical parameters. We interpreted these results as a Poissonic noise because the expected value equal the variance for the Poissonic distribution. We showed that these physical parameters (fluxes, fluences and durations) could discriminate between the spectral classes and the peak flux type spectra was better separable.

Acknowledgements This work was supported by the Hungarian OTKA NN-111016 grant and the ÚNKP-17-3 New National Excellence Program of the Ministry of Human Capacities.

\section{References}

[1] P. Kumar and B. Zhang, The physics of gamma-ray bursts and relativistic jets, Phys. Rep. 561 (Feb., 2015) 1-109, [1410.0679].

[2] J. P. Norris, T. L. Cline, U. D. Desai and B. J. Teegarden, Frequency of fast, narrow gamma-ray bursts, Nature 308 (Mar., 1984) 434.

[3] I. Horváth, A Third Class of Gamma-Ray Bursts?, ApJ 508 (Dec., 1998) 757-759, [astro-ph/9803077]. 
[4] S. Mukherjee, E. D. Feigelson, G. Jogesh Babu, F. Murtagh, C. Fraley and A. Raftery, Three Types of Gamma-Ray Bursts, ApJ 508 (Nov., 1998) 314-327, [astro-ph/ 9802085 ].

[5] I. Horváth, A further study of the BATSE Gamma-Ray Burst duration distribution, A\&A 392 (Sept., 2002) 791-793, [astro-ph/ 0205004 ].

[6] I. Horváth, L. G. Balázs, Z. Bagoly, F. Ryde and A. Mészáros, A new definition of the intermediate group of gamma-ray bursts, A\&A 447 (Feb., 2006) 23-30, [astro-ph/ 0509909 ].

[7] I. Horváth, L. G. Balázs, Z. Bagoly and P. Veres, Classification of Swift's gamma-ray bursts, A\&A 489 (Oct., 2008) L1-L4, [0 808.1067].

[8] I. Horváth, Classification of BeppoSAX's gamma-ray bursts, Ap\&SS 323 (Sept., 2009) 83-86, [0905.0860].

[9] P. Veres, Z. Bagoly, I. Horváth, A. Mészáros and L. G. Balázs, A Distinct Peak-flux Distribution of the Third Class of Gamma-ray Bursts: A Possible Signature of X-ray Flashes?, ApJ 725 (Dec., 2010) 1955-1964, [1010.2087].

[10] A. de Ugarte Postigo, I. Horváth, P. Veres, Z. Bagoly, D. A. Kann, C. C. Thöne et al., Searching for differences in Swift's intermediate GRBs, A\&A 525 (Jan., 2011) A109, [1006.4469].

[11] I. Horváth and B. G. Tóth, The duration distribution of Swift Gamma-Ray Bursts, Ap\&SS 361 (May, 2016) $155,[1604.00887]$.

[12] M. Tarnopolski, Analysis of gamma-ray burst duration distribution using mixtures of skewed distributions, MNRAS 458 (May, 2016) 2024-2031, [1506.07801].

[13] I. Horváth, J. Hakkila and Z. Bagoly, Possible structure in the GRB sky distribution at redshift two, A\&A 561 (Jan., 2014) L12, [1401.0533].

[14] I. Horváth, Z. Bagoly, J. Hakkila and L. V. Tóth, New data support the existence of the Hercules-Corona Borealis Great Wall, A\&A 584 (Dec., 2015) A48, [1510.01933].

[15] L. G. Balázs, L. Rejtô and G. Tusnády, Some statistical remarks on the giant GRB ring, MNRAS 473 (Jan., 2018) 3169-3179, [1710.01621].

[16] L. G. Balázs, Z. Bagoly, J. E. Hakkila, I. Horváth, J. Kóbori, I. I. Rácz et al., A giant ring-like structure at $0.78<z<0.86$ displayed by GRBs, MNRAS 452 (Sept., 2015) 2236-2246, [1507.00675].

[17] Z. Bagoly, D. Szécsi, L. G. Balázs, I. Csabai, I. Horváth, L. Dobos et al., Searching for electromagnetic counterpart of LIGO gravitational waves in the Fermi GBM data with $A D W O, A \& A$ 593 (Sept., 2016) L10, [1603.06611].

[18] D. Gruber, A. Goldstein, V. W. von Ahlefeld, P. N. Bhat, E. Bissaldi, M. S. Briggs et al., The fermi gbm gamma-ray burst spectral catalog: Four years of data, The Astrophysical Journal Supplement Series 211 (2014) 12.

[19] P. N. Bhat, C. A. Meegan, A. von Kienlin, W. S. Paciesas, M. S. Briggs, J. M. Burgess et al., The third fermi gbm gamma-ray burst catalog: The first six years, The Astrophysical Journal Supplement Series 223 (2016) 28.

[20] R. A. Fisher, The use of multiple measurements in taxonomic problems, Annals of Eugenics 7 (1936) 179-188.

[21] I. I. Racz, L. G. Balázs, I. Horvath, et al.MNRAS, submitted for publication (2017) .

[22] R. D. Yates and D. Goodman, Probability and Stochastic Processes: A Friendly Introduction for Electrical and Computer Engineers. Wiley, October, 2014. 